\title{
Immunohistochemical comparative study of the odontogenic keratocysts and other odontogenic lesions
}

\author{
Juan-Carlos de-Vicente ${ }^{1}$, Aintzane Torre-Iturraspe ${ }^{1}$, Ana-María Gutiérrez ${ }^{2}$, Paloma Lequerica-Fernández ${ }^{3}$ \\ ${ }^{1}$ Department of Oral and Maxillofacial Surgery (Head: Prof. Dr. Juan Sebastián López-Arranz, M.D., Ph.D.) \\ ${ }^{2}$ Department of Pathology (Head: Prof. Dr. Manuel F. Fresno, M.D., Ph.D.) \\ ${ }^{3}$ Instituto Universitario de Oncología del Principado de Asturias (Head: Prof. Dr. Carlos Suárez-Nieto, M.D., Ph.D.). Univer- \\ sity Central Hospital of Asturias and Faculty of Medicine, Oviedo, Asturias, Spain
}

\author{
Correspondence: \\ Servicio de Cirugía Oral y Maxilofacial \\ Hospital Universitario Central de Asturias \\ C/ Celestino Villamil $s / n$. \\ 33006. Oviedo. Asturias, \\ Spain \\ jvicente@.uniovi.es
}

Received: $13 / 09 / 2009$

Accepted: $22 / 01 / 2010$

\author{
de Vicente JC, Torre-Iturraspe A, Gutiérrez AM, Lequerica-Fernández P. \\ Immunohistochemical comparative study of the odontogenic keratocysts \\ and other odontogenic lesions. Med Oral Patol Oral Cir Bucal. 2010 Sep \\ 1;15 (5):e709-15. \\ http://www.medicinaoral.com/medoralfree01/v15i5/medoralv15i5p709.pdf

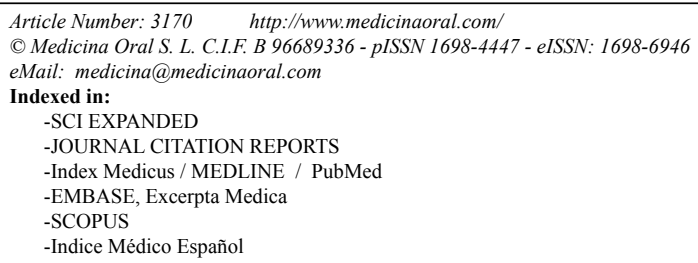

\begin{abstract}
Objectives: The present study was undertaken to compare the pattern of expression of EGFR, cyclin D1, Ki-67, p-53 and carcinoembryonic antigen (CEA) in the epithelial lining of odontogenic keratocysts, dentigerous cysts, radicular cysts and ameloblastomas.

Methods: four micrometers, formalin-fixed, paraffin-embedded tissue sections from 11 odontogenic keratocysts, 10 dentigerous cysts, 10 radicular cysts and 10 ameloblastomas were immunohistochemically studied.

Results: There were statistically significant differences between cyclin D1 expression in odontogenic keratocysts and radicular cysts $(p=0.001)$ and ameloblastomas $(p=0.04)$. The differences in CEA expression between the four studied lesions were statistically significant $(p<0.0005)$. Proliferating cells were significantly more prevalent in odontogenic keratocysts $(p<0.0005)$ with a mean percentage of Ki-67 positively stained nuclei of $40 \%$. In dentigerous cysts this mean was of $17 \%$, of $15.5 \%$ in RC and of 7.8 in ameloblastomas.

Conclusion: Some of these findings could support the theory that odontogenic keratocysts are neoplastic in origin, but other results clearly support that these lesions are developmental cysts with some neoplastic properties because of the high intrinsic growth potential.
\end{abstract}

Key words: Odontogenic keratocysts, CEA, EGFR, cyclin D1, Ki-67, p53. 


\section{Introduction}

Different types of odontogenic cysts and tumors arise from derivates of embryologic dental lamina. The potential for further proliferation of these epithelial remnants during formation of a cyst is different and thus lead to variations in their molecular expression and biological behaviour, due to an underlying mechanism that remains highly speculative. Three major categories of odontogenic cysts are usually distinguished: radicular cysts (RCs), follicular or dentigerous cysts (DCs), and keratocysts. Keratocysts accounts for 3\% to $11 \%$ of odontogenic cysts (1) and it is well known that they show aggressive behaviour with higher rates of recurrence than other types of odontogenic cysts, as well as a tendency to invade adjacent tissue (1). Odontogenic keratocysts (OKCs) can arise sporadically or, about 4 to $5 \%$ of them (2), in association with the nevoid basal cell carcinoma syndrome (Gorlin syndrome) in which case the clinical behaviour of OKCs is even more aggressive. This aggressive biological behavior of the OKCs and its tendency to recurrence might be associated with cell kinetics in the lining epithelium. Numerous reports focusing on the greater proliferative potential of the epithelial lining of OKCs, showing a high mitotic index (3) compared with other types of odontogenic cysts. Consequently, it has given rise to the hypothesis that odontogenic keratocysts may represent benign neoplasms instead of odontogenic cysts.

Cell kinetics is under the influence of growth factors such as the peptide Epidermal Growth Factor (EGF) and its receptor (EGFR). EGFR distribution is observed in normal epithelium, mainly in proliferating basal cells, and is expressed most intensely in both benign and malignant epithelial neoplasms. Interestingly, EGF and EGFR might provide epigenetic control of odontogenesis by modulating cell growth and cell interaction (4). Since the majority of human neoplasms are characterized by an imbalance of the regulatory cell cycle control process, the study of proto-oncogenes and tumor suppressor genes, such as cyclin D1 and p53, involved in critical check-points of cell growth could improve our knowledge about the biological substrate of OKCs behaviour. Ki-67 antigen is a protein expressed in all phases of the cell cycle, except in G0. Its expression increases with cell cycle progression, rising during the latter half of the S phase, reaching a peak in the G2 and $\mathrm{M}$ phase and rapidly decreasing after mitosis. Thus Ki67 expression informs about the number of proliferating cells in a tissue.

Tumor markers, usually proteins, are produced by the body in response to a tumor or by the tumor tissue itself. The tumor marker carcinoembryonic antigen (CEA) has been detected in many cancers, as well as in odontogenic tumors and keratocysts (5), but its significance in the latter is unclear. All of the above mentioned gene products have been studied in odontogenic keratocysts, and have been related to their aggressive nature, but the results are inconclusive.

Based on the hypothesis that odontogenic keratocysts are true neoplasms, the present study was undertaken to characterize the pattern of expression of EGFR, cyclin D1, Ki-67, p-53 and CEA in the epithelial lining of odontogenic keratocysts, and two other cysts and a true neoplasm, dentigerous cysts, radicular cysts and ameloblastomas, respectively.

\section{Material and Methods}

\section{Tissue samples}

After reviewing clinical information and histology, cases of parakeratinized odontogenic keratocyst $(\mathrm{n}=$ $11)$, dentigerous cyst $(n=10)$, radicular cyst $(n=10)$ and ameloblastoma $(n=10)$ were selected from the files of the Departments of Oral and Maxillofacial Surgery and Pathology, Hospital Universitario Central de Asturias, Spain, from January 1994 to July 2000. Inclusion criteria were surgical treatment performed according to standard procedures and consisting of the resection of the cyst or tumor, complete clinical and histopathologic data and availability of sufficient tissue material. None of the odontogenic keratocysts were associated with the nevoid basal cell carcinoma syndrome.

\section{Antibodies}

Antibodies used in this study are listed in (Table 1).

Immunohistochemical study

All selected cases had been routinely fixed in 10\% neutral formalin ( $\mathrm{pH} 7.4$ ) for $72 \mathrm{~h}$ and embedded in paraffin.

Table 1. Antibodies employed in this study.

\begin{tabular}{|l|l|c|c|l|}
\hline Antibodies & \multicolumn{1}{|c|}{ Source } & Clones & Dilutions & \multicolumn{1}{c|}{ Specificity } \\
\hline EGFR & Dako, Carpinteria, CA & H11 & $1: 50$ & Transmembranous receptor of EGFR \\
\hline CEA & Dako, Glostrup, Denmark & II-7 & $1: 50$ & Carcinoembryonic antigen \\
\hline p53 & Dako, Carpinteria, CA & D07 & $1: 100$ & P53 protein \\
\hline Cyclin D1 & Neo Markers & SP4 & $1: 50$ & Cyclin D1 \\
\hline Ki67 & Dako, Carpinteria, CA & MIB-1 & $1: 100$ & Nuclear antigen Ki-67 \\
\hline
\end{tabular}

All these antibodies are Mouse monoclonal with the only exception of Cyclin D1 that is rabbit polyclonal. 
In ameloblastomas, decalcification of the specimen was performed in $10 \%$ formic acid for 48 to $72 \mathrm{~h}$. Four- $\mu \mathrm{m}-$ thick tissue sections were mounted on poly-L-lysinecoated slides and treated in a routine way. The sections were then heated in $10 \mathrm{mM}$ citrate buffer $(\mathrm{pH} 6.5)$ for 4 min in a microwave oven at $500 \mathrm{~W}$ to retrieve antigenicity. Non-specific conjugation was blocked with a solution of $20 \%$ rabbit serum (DAKO, Glostrup, Denmark) applied to the sections for $10 \mathrm{~min}$.

Immunohistochemistry was performed incubating the sections with primary antibodies against EGFR, cyclin D1, Ki-67, p53 and CEA for 30 minutes at room temperature. They were then rinsed in PBS, and bound antibody was detected using EnVision polymer technology (K4001, DAKO, Carpinteria, CA) for 30 min according to the manufacturer's instructions. After washing in PBS, staining was developed with 3, 3'-diaminobenzidine-tetrahydrochloride in 50mM Tris-HCL ( $\mathrm{pH} 7.5$ ) containing $0.001 \%$ hydrogen peroxide for $5 \mathrm{~min}$, and then lightly counterstained with Mayer's hematoxylin. All slides were independently reviewed by two investigators.

The EGFR expression was scored varying from 0 to $2+$ ( 0 was defined as being negative and $1+$ and $2+$ as being positive and intense, respectively). Staining for Ki-67 was measured as the percentage of positively stained nuclei (proliferation index) in 200 epithelial cells in a consecutive field. We scored the cyclin D1 and p53 expression according to three categories: negative (absence of staining) and positive, and this latter into two additional categories, focal or diffuse staining. Finally, CEA expression was assessed as negative or positive.

Statistical analysis

Analysis of the data was performed using SPSS software, version 14.0. The $\chi 2$ test was used for comparisons of categorical variables and the Kruskal-Wallis test for continuous variables. Two-sided $\mathrm{p}$ values are reported at a significance level of $p$ equal or less than 0.05 .

\section{Results}

The expression of EGFR, CEA, p53 and cyclin D1 is summarized in (Table 2). EGFR expression was positive in $100 \%$ of ameloblastomas, $73 \%$ of the OKCs, $40 \%$ of dentigerous cysts and $30 \%$ of radicular cysts. In nine cases of ameloblastomas EGFR stain was diffuse, while in one case the reaction was focally located. In odontogenic keratocysts EGFR immunostaining was located in the basal cell layer, and was focal in eight and diffuse in no one case (Fig. 1). In all positive dentigerous and radicular cysts, immunostaining was focal. In all positive specimens cytochemical localization of EGFR was of the cell membrane positive type.

Immunostaining for cyclin D1 was positive in $50 \%$ of DCs, $30 \%$ of RCs, $40 \%$ of ameloblastomas, and $91 \%$ of OKCs. In almost all OKCs, cyclin D1 was expressed in a focal parabasal pattern (Fig. 2A), while in DCs and in $\mathrm{RCs}$ focal and diffuse patterns were equally observed. In all positive ameloblastomas, the staining pattern was only diffuse. P53 immunoexpression was positive in $64 \%$ of OKCs, $40 \%$ of DCs and RCs, and $30 \%$ of amelo-

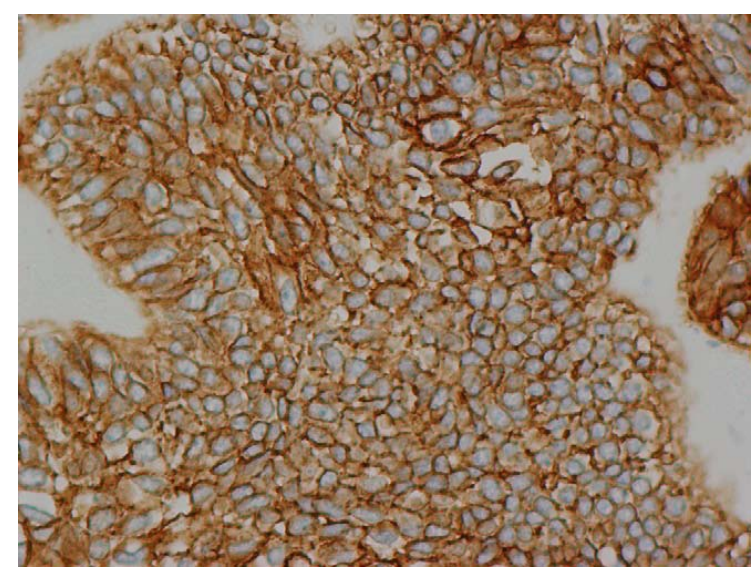

Fig. 1. Positive expression of EGFR in an odontogenic keratocist. Staining is located to the cell membrane and extending from basal to superficial cells in cyst lining (Original magnification, $\mathrm{x} 400$ ).

Tabla 2. EGFR, Cyclin D1, P53 and CEA staining [number and percentage] in odontogenic keratocysts (OKS), dentigerous cysts (DCs), radicular cysts (RCs), and ameloblastomas (Am).

\begin{tabular}{|l|c|c|c|c|c|c|c|c|c|c|c|}
\hline \multirow{2}{*}{ Lesion } & \multicolumn{3}{|c|}{ EGFR } & \multicolumn{4}{c|}{ Cyclin D1 } & \multicolumn{3}{c|}{ P53 } & \multicolumn{2}{c|}{ CEA } \\
\cline { 2 - 12 } & $0(\%)$ & $1+(\%)$ & $2+(\%)$ & $0(\%)$ & Focal (\%) & Difuso (\%) & $0(\%)$ & Focal (\%) & Difuso (\%) & $-(\%)$ & $+(\%)$ \\
\hline OKCs & $3(27)$ & $8(73)$ & $0(0)$ & $1(9)$ & $9(82)$ & $1(9)$ & $4(36)$ & $6(55)$ & $1(9)$ & $0(0)$ & $11(100)$ \\
\hline DCs & $6(60)$ & $4(40)$ & $0(0)$ & $\begin{array}{c}5 \\
(50)\end{array}$ & $3(30)$ & $2(20)$ & $6(60)$ & $2(20)$ & $2(20)$ & $7(70)$ & $3(30)$ \\
\hline RCs & $7(70)$ & $3(30)$ & $0(0)$ & $\begin{array}{c}7 \\
(70)\end{array}$ & $2(20)$ & $1(10)$ & $6(60)$ & $2(20)$ & $2(20)$ & $10(100)$ & $0(0)$ \\
\hline Am & $0(0)$ & $1(10)$ & $9(90)$ & $\begin{array}{c}6 \\
(60)\end{array}$ & $4(40)$ & $0(0)$ & $7(70)$ & $3(30)$ & $0(0)$ & $10(100)$ & $0(0)$ \\
\hline
\end{tabular}

EGFR: Am vs OKCs, DCs, y RCs: $p<0,0005$. Cyclin D1: OKC vs DCs: $p=0,08$; OKCs vs RCs: $p=0,01$; OKCs vs Am: $p=0,04$. CEA: OKCs vs DCs; OKCs vs RCs y OKCs vs Am: p<0,0005. 


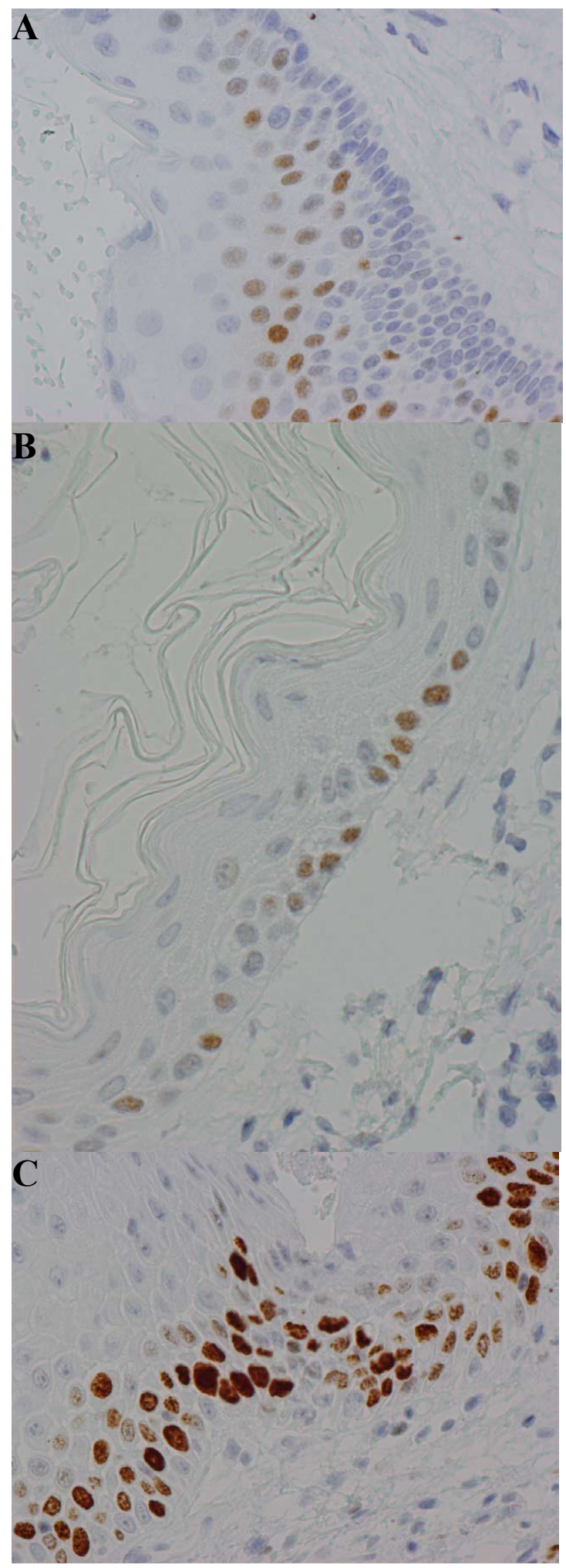

Fig. 2. Immunohistochemical expression of cell-cycle-related factors in lining epithelium of odontogenic keratocysts. (A) Positive staining for cyclin D1 in the nuclei of parabasal cells. (B) Reactivity for $\mathrm{p} 53$ in the nuclei of basal cells. (C) Immunostaining for Ki-67. The Ki-67 immunopositive cells can be detected in the basal and intermediate layers of the lining epithelium of cyst (Original magnification, $\mathrm{x} 400$ ). blastomas. Immunolabelling for P53 was observed in the nuclei of cells scattered in the whole lining epithelium of OKCs, but mainly basally (Fig. 2B). In DCs and in RCs, P53 protein immunoexpression was located in the basal cell layer, and in ameloblastomas its expression was scattered and only occasionally clustered. Almost all cases (19 of 20) of OKCs and only three of ten dentigerous cysts were immunoreactive with CEA antibody. On the other hand, no cases of radicular cysts or ameloblastomas showed CEA immunoreactivity. CEA immunoreactive cells showed two basic patterns of stain: diffuse staining throughout the cytoplasm and peripheral staining along the cell membrane (Fig. 3). Neither OKCs nor other two dentigerous cysts showed any staining within basal and parabasal epithelial cell layers. Conversely, CEA reactivity was consistently detected in mature cells located in superficial layers and was diffuse in all OKCs. In the three immunopositive dentigerous cysts the stain was also superficial and focal.

EGFR was significantly more common in ameloblastomas than in the three types of studied odontogenic cysts, being statistically significant differences with odontogenic keratocysts $(p<0.0005)$. There were statistically significant differences between cyclin D1 expression in OKCs and in RCs $(\mathrm{p}=0.01)$ and ameloblastomas $(\mathrm{p}=0.04)$. The differences in CEA expression between the four studied lesions were statistically significant $(\mathrm{p}<0.0005)$, with the only exception of radicular cysts and ameloblastomas that not were significantly different $(p=0.07)$. There were no significant differences between immunoexpression of P53 among the studied lesions(Table 2).

Proliferating cells, detected by positive nuclear staining with Ki67, were most prevalent in OKCs with a mean percentage of positively stained nuclei of $40 \%$ (Figure 2 C). In dentigerous cysts this mean was of $17 \%$, of $15.5 \%$ in radicular cysts and of $7.8 \%$ in ameloblastomas. In odontogenic keratocysts, Ki-67 staining was speckled and especially localized in the nuclei of basal and suprabasal cells. The cell proliferation index was significantly higher in OKCs than in the other lesions $(p<0.0005)$.

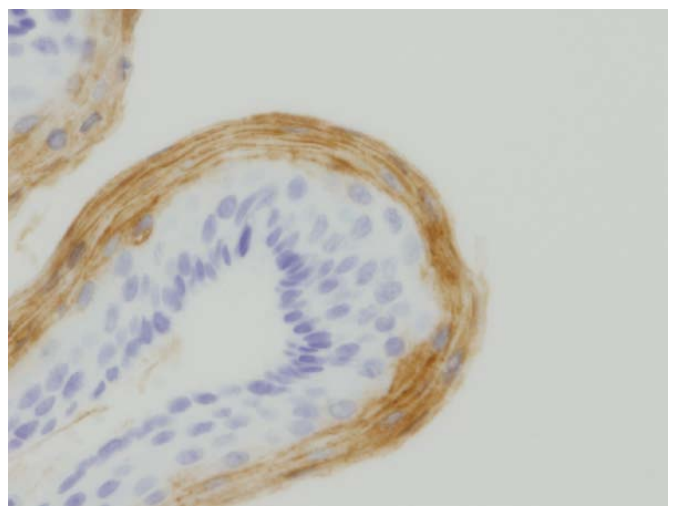

Fig. 3. Positive staining for CEA in the epithelium of an odontogenic keratocyst (Original magnification, $\mathrm{x} 400$ ). 


\section{Discussion}

The classification of odontogenic jaw cysts is based on their histology, pathogenesis and presumed developmental origin. Radicular cysts have an epithelial lining derived from the epithelial rests of Malassez, follicular cysts developing by passive pooling of fluid beneath the crown follicle in association with impeded tooth eruption and their epithelium is derived from reduced enamel epithelium. It is presumed that epithelia of OKCs originates from the odontogenic epithelium of the dental lamina or its remnants, prior to tooth formation. However, it has also been suggested that odontogenic keratocysts could be derived from basal cells of the oral mucosa (6). Odontogenic keratocysts show a particular tendency to recur after their surgical removal that could be due to a multilocular nature or a presence of daughter or satellite cysts that are left behind. However, it has also been hypothesized that the epithelium of these cysts could have an intrinsic potential to growth, resembling benign neoplasms (7). Ameloblastomas are tumors that arise from rests of the dental lamina. Thus, OKCs and ameloblastomas derived from the same cell population, and like ameloblastomas, OKCs may behave aggressively and can penetrate cortical bone, extending into the surrounding tissues, with also they show recurrence rates ranging from $3 \%$ to $60 \%$ (8). The EGF, a potent mitogen for epithelial cells, is important in the regulation of proliferation in normal and neoplastic cells. EGF acts on target cells by binding to a specific cell surface receptor (EGFR) thereby stimulating a cellular protein kinase which catalyses the phosphorylation of tyrosine. This is the first step in a chain of reactions that culminate in mitosis (9). In this study, we have observed a trend of decreased staining positivity for EGFR in the following order: ameloblastomas (100\%), OKCs (73\%), dentigerous cysts $(40 \%)$ and radicular cysts $(30 \%)$. These results demonstrated that the epithelial cells of the aforementioned lesions express EGFR in variable patterns. In particular ameloblastomas and developmental odontogenic cysts (OKCs and dentigerous cysts) exhibited more intense staining for EGFR than those of inflammatory radicular cysts that could probably be related with the biological aggressiveness of ameloblastomas and OKCs. These results are in agreement with that of Li et al. (10), which found that ameloblastomas, odontogenic keratocysts, dentigerous cysts and radicular cysts were positive for EGFR, but contrast with Shrestha et al. (11), who separated primordial cysts from keratocysts for not explained reasons, and observed that $60 \%$ and $75 \%$, respectively, showed a positive EGFR reaction. Furthermore, the EGFR immunopositivity observed in dentigerous cysts and radicular cysts was of $47.4 \%$ and $35 \%$, respectively, and in clear constrast with our results, they did not find EGFR positivity in any of 21 ameloblastomas studied. The transition between different cell cycle stages is regulated at several checkpoints. Regulation of the G1-S transition is controlled by the $\mathrm{Rb}$ pathway proteins, which include, among others, the cyclin D1 gene. The overexpression of this protein shows accelerated G1 progression entering in the S-phase of cell cycle, with lower cell dependence on growth factors for proliferation. Cyclin D1 may play an important role in tumorigenesis and it has been detected in malignant tumors but also in benign neoplasms. In the present study cyclin D1 was found in $91 \%$ of OKCs, and the rate of its expression was significantly higher than that of dentigerous cysts, radicular cysts and ameloblastomas. Conversely, Lo Muzio et al. (2) have found cyclin D1 overexpression in all OKCs associated with the Gorlin syndrome, while all sporadic odontogenic keratocysts on their study were negative. This difference, as well as we have observed with EGFR, is likely to be explained by differences in method. Regarding cyclin D1 expression, Lo Muzio et al. (2) used the clone 5D4, diluted 1:800, while we used the clone SP4 at a 1:50. We observed the cyclin D1 expression in a focal and basal pattern, as was also seen in dentigerous cysts and in radicular cysts. In all positive ameloblastomas, the staining pattern was only diffuse.

The mitotic index, immunohistochemically measured as $\mathrm{Ki}-67$ positive cells, is greater in OKCs lining epithelium than in dentigerous and radicular cysts, and is similar to those seen in ameloblastoma or in actively growing dental lamina $(12,13)$. These differences in the proliferative activity of the OKCs epithelium are considered suggestive of an intrinsic growth potential that could play a role in its biological behaviour $(13,14)$. Similarly, an increased number of P53 positively stained cells have been found in the epithelium of OKCs in comparison with dentigerous cysts and radicular cysts and its overexpression has been thought to result from overproduction and/or stabilization of wild type P53 protein due to the increased proliferation of the epithelial cells (15). We have found that $\mathrm{Ki}-67$ staining was principally speckled and especially localized in the nuclei of basal and suprabasal cells, which imply that cells of these layers posesses the highest proliferative activity in OKCs. In this study the cellular proliferation, measured by the expression of $\mathrm{Ki}-67$, is higher in OKCs than in dentigerous ones. These findings suggest that in OKCs could exist a defective tumor suppression function, that is reflected in a proliferative activity higher than in ameloblastomas (p53 expression in odontogenic keratocysts and ameloblastomas was of $64 \%$ and $30 \%$, respectively), as could be seen in the epithelial linings of the former.

On the other hand, a high proliferative index in OKCs could also be explained by the presence of inflammation in response of an infection or traumatism (16). Furthermore, this high proliferative activity could decrease when inflammation decreases. 
The p53 gene, located at the short arm of chromosome 17 is the best known tumor suppressor gene, and p53 abnormalities are the most common genetic abnormalities in human neoplasms. Wild-type p53 protein acts as a DNA-binding transcription factor and its activation elicits adaptative responses including growth arrest and apoptosis. Alterations of the p53 gene or inactivation of its protein product are usually involved in carcinogenesis, and tissues that express p53 seem to have a higher proliferative activity than p53-negative epithelia. Ogden et al. (17) found overexpression of p53 in odontogenic keratocysts associated with the nevoid basal cell carcinoma syndrome, but studies correlating p53 and clinicopathological parameters in oral cysts have yielded different results. P53 protein has, in normal tissues, a very short half-life and cannot be detected with immunohistochemical techniques, whereas the p53 mutation produces an increased stability. Mutation of p53 gene is a common finding in a wide range of human tumors and usually results in a more stable protein that accumulates in cells, allowing it to be detected immunohistochemically. Agaram et al. (8) found loss of heterozygosity in 7 of $10 \mathrm{OKCs}$, being p53 one of the genes that most frequently exhibited allelic losses (66\%). However, mutation of p53 gene is not only the reason why P53 protein remains longer in cells and can be detected. In addition, defects in protein degradation and binding to other proteins, such as SV40, E1B, or HPVE6 viral proteins can also stabilize p53 protein in cells (18).

In this study, p53 immunoexpression was positive in $50 \%$ of $\mathrm{OKCs}, 40 \%$ of dentigerous cysts and radicular cysts, and $30 \%$ of ameloblastomas, but without statistically significant differences among them. Conversely, Piatelli et al. (19), also found a higher p53 expression in odontogenic keratocysts than in dentigerous and radicular cysts, and differences between the three groups were stastistically significant. Ogden et al. (17) using CM-1 antibody reported that 5 of 12 odontogenic keratocysts expressed P53, while Lombardi et al. (20) with the same clone CM-1 found p-53 in 50\% of their OKCs, including non-recurrent, recurrent and associated with the basalcell nevus syndrome. Piatelli et al. (19), employing the clone DO7 found p-53 expression in $45 \%$ of 20 odontogenic keratocysts. We have found the same percentage of positively stainded cells in OKCs as these authors on the contrary we have found higher positivity rates in dentigerous cysts and radicular cysts. It is not clear why benign lesions such as OKCs should express p53 protein at an immunohistochemically detectable level, but an expression of p53 for physiological reasons, such as protein's normal function in DNA repair, could not be excluded. However, Slootweg (15) has found that ameloblastomas and odontogenic keratocysts presented a dense staining, whereas radicular and dentigerous cysts were faintly stained or negative, and he hypothesized that cell
P53 positively stained could be related to increased proliferation. Taken together, the high frequency of P53 expression and the higher mitotic activity in the epithelial lining of odontogenic keratocysts correlates well, and at least partially could explain the aggressive behaviour of odontogenic keratocysts consistent with that of benign tumors.

Howell et al. (5) studied the immunoexpression of carcinoembryonic antigen (CEA) in 7 odontogenic keratocysts and in 19 ameloblastomas. They found that all cases of odontogenic keratocysts and $68 \%$ of ameloblastomas showed CEA immunoreactivity. Similarly, we have observed CEA immunoexpression in all OKCs, but conversely, none of our ameloblastomas showed reaction against CEA. Howell et al. (5) stated that a possible explanation for CEA positive reaction could be due to a nonspecific cross-reaction with keratin. However, we have also studied the immunoexpression of pancytokeratin (Clones AE1/AE3, Dako, Carpinteria; dilution: 1:400) in our OKCs and ameloblastomas and observed an immunoexpression both in all odontogenic keratocysts and ameloblastomas (data not shown). Thus, the CEA expression probably reflects the aggressive nature of odontogenic keratocysts.

The immunohistochemical findings in the lesions investigated in the present study suggest that the biological behaviour of OKCs may be associated with deregulation of cell cycle in the lining epithelium, indicated by increased expression of EGFR, cyclin D1, cell proliferation and CEA. This supports the neoplastic nature of OKCs. However, they have shown important differences with the only neoplasm studied here, i.e. ameloblastomas. Thus, while $100 \%$ of the ameloblastoma cases reacted positively with EGFR, only $73 \%$ of odontogenic keratocysts did. On the other hand, the reverse was true in the case of cyclin D1, 91\% of the cases of OKCs reacted positively and only $40 \%$ of ameloblastomas were reactive. Moreover, CEA stain was positive in 100\% of OKCs cases but in none of the ameloblastomas. Furthermore, our results also show a fundamental difference between dentigerous and radicular cysts on the one hand and $\mathrm{OKCs}$ on the other hand in respect of their growth factor requirements and cell's cycle control. Some of the findings of this study could contribute to support the neoplastic origin of OKCs. However, other results clearly revealed that odontogenic keratocysts have also good resemblance with developmental odontogenic disorders as dentigerous and radicular cysts. Therefore, from an immunohistochemical point of view, OKCs could be seen as odontogenic neoplasms that show similarities with odontogenic cysts or as developmental cysts with some neoplastic properties because of the high intrinsic growth potential. 


\section{References}

1. Chirapathomsakul D, Sastravaha P, Jansisyanont P. A review of odontogenic keratocysts and the behavior of recurrences. Oral Surg Oral Med Oral Pathol Oral Radiol Endod. 2006;101:5-9.

2. Lo Muzio L, Staibano S, Pannone G, Bucci P, Nocini PF, Bucci $\mathrm{E}$, et al. Expression of cell cycle and apoptosis-related proteins in sporadic odontogenic keratocysts and odontogenic keratocysts associated with the nevoid basal cell carcinoma syndrome. J Dent Res. 1999; 78:1345-53

3. Scharffetter K, Balz-Herrmann C, Lagrange W, Koberg W, Mittermayer C. Proliferation kinetics-study of the growth of keratocysts. Morpho-functional explanation for recurrences. J Craniomaxillofac Surg. 1989;17:226-33.

4. Partanen AM, Thesleff I. Localization and quantitation of 125Iepidermal growth factor binding in mouse embryonic tooth and other embryonic tissues at different developmental stages. Dev Biol. 1987;120:186-97.

5. Howell RE, Handlers JP, Aberle AM, Abrams AM, Melrose RJ. CEA immunoreactivity in odontogenic tumors and keratocysts. Oral Surg Oral Med Oral Pathol. 1988;66:576-80.

6. Stoelinga PJ, Peters JH. A note on the origin of keratocysts of the jaws. Int J Oral Surg. 1973;2:37-44.

7. Hormia M, Ylipaavalniemi P, Nagle RB, Virtanen I. Expression of cytokeratins in odontogenic jaw cysts: monoclonal antibodies reveal distinct variation between different cyst types. J Oral Pathol. 1987;16:338-46.

8. Agaram NP, Collins BM, Barnes L, Lomago D, Aldeeb D, Swalsky $\mathrm{P}$, et al. Molecular analysis to demonstrate that odontogenic keratocysts are neoplastic. Arch Pathol Lab Med. 2004;128:313-7.

9. Carpenter G. Epidermal growth factor: biology and receptor metabolism. J Cell Sci Suppl. 1985;3:1-9.

10. Li TJ, Browne RM, Matthews JB. Expression of epidermal growth factor receptors by odontogenic jaw cysts. Virchows Arch A Pathol Anat Histopathol. 1993;423:137-44.

11. Shrestha P, Yamada K, Higashiyama H, Takagi H, Mori M. Epidermal growth factor receptor in odontogenic cysts and tumors. $\mathrm{J}$ Oral Pathol Med. 1992;21:314-7.

12. Matthews JB, Mason GI, Browne RM. Epithelial cell markers and proliferating cells in odontogenic jaw cysts. J Pathol. 1988;156:28390.

13. Li TJ, Browne RM, Matthews JB. Epithelial cell proliferation in odontogenic keratocysts: a comparative immunocytochemical study of Ki67 in simple, recurrent and basal cell naevus syndrome (BCNS)-associated lesions. J Oral Pathol Med. 1995;24:221-6.

14. El Murtadi A, Grehan D, Toner M, McCartan BE. Proliferating cell nuclear antigen staining in syndrome and nonsyndrome odontogenic keratocysts. Oral Surg Oral Med Oral Pathol Oral Radiol Endod. 1996;81:217-20.

15. Slootweg PJ. p53 protein and Ki-67 reactivity in epithelial odontogenic lesions. An immunohistochemical study. J Oral Pathol Med. 1995;24:393-7.

16. De Paula AM, Carvalhais JN, Domingues MG, Barreto DC, Mesquita RA. Cell proliferation markers in the odontogenic keratocyst: effect of inflammation. J Oral Pathol Med. 2000;29:477-82.

17. Ogden GR, Chisholm DM, Kiddie RA, Lane DP. p53 protein in odontogenic cysts: increased expression in some odontogenic keratocysts. J Clin Pathol. 1992;45:1007-10.

18. Nylander K, Dabelsteen E, Hall PA. The p53 molecule and its prognostic role in squamous cell carcinomas of the head and neck. $\mathrm{J}$ Oral Pathol Med. 2000;29:413-25.

19. Piattelli A, Fioroni M, Santinelli A, Rubini C. P53 protein expression in odontogenic cysts. J Endod. 2001;27:459-61.

20. Lombardi T, Odell EW, Morgan PR. p53 immunohistochemistry of odontogenic keratocysts in relation to recurrence, basal-cell budding and basal-cell naevus syndrome. Arch Oral Biol. 1995;40:1081-4.

\section{Acknowledgements}

The auhors thank to Dr. Jonas Hannestad (Department of Psychiatry, Duke University) for help in reviewing the manuscript for English syntax. 